CAMBRIDGE, MASSACHUSETTS, USA, 13 - 15 October, 2020

\title{
Matrix-Based Landscapes for Communication between Market and Product Perspective
}

\author{
Michael Riesener ${ }^{1}$, Christian Dölle ${ }^{1}$, Benjamin Lender $^{1}$, Günther Schuh ${ }^{1}$ \\ ${ }^{1}$ Laboratory for Machine Tools and Production Engineering WZL of the RWTH Aachen
}

\begin{abstract}
In the development of cyber-physical systems, communication between teams representing market and product perspective is often marked by different incentives. The market perspective, representing the customer, argues for variability and individualization, while the product perspective argues for the simplest, most efficient and least expensive product variant. There is a need for balancing individualization with cost effective development and production. This paper discusses the use of matrices as means to create transparency for both market and product perspective, showing where individualization is both most attractive to the customer and least expensive. In doing so, the framework delivers an approach to a key strategic problem: optimizing the efficiency of internal and external complexity by visual representation of individualization versus standardization potentials.
\end{abstract}

Keywords: variant management, complexity management, project management, DSM, standardization

\section{Introduction}

Current trends of mass individualization put companies under increasing pressure of managing variants and offering a wide portfolio while remaining cost effective (Fuchs and Golenhofen, 2019). They describe customers being increasingly heterogeneous and markets swaying from seller- to buyer-driven markets. Increased variability expands the internal complexity, raising costs of the development process and binding resources in the company. Vangheluwe (2018) underlines the relevance of this to cyber-physical systems, whose increase in complexity has been accelerating in recent years. Schuh (2013) as well as Alptekinoğlu and Corbett (2008) name variant management as an approach to dealing with this increasingly dynamic market situation. On the one hand, these trends put pressure on the team representing the market perspective to argue for an increase in variability, offering each customer precisely the solution the customer may be looking for (Crawley et al., 2016). The product perspective's goal on the other hand is developing products and variants cost-effectively, which implies using commonalities and reducing exotic variants (Anderson, 1995; Schuh and Riesener, 2017). It may not be possible to eliminate this discrepancy in all cases, yet it is possible to better align the two perspectives. Identifying where variability is most and least expensive offers a tool to both market and product perspective to advance their objectives. This paper aims at identifying, which cases of individualization offer the highest potential to be both attractive to the customer and reasonably viable for a cost-effective development. The term landscape is used to describe a graph of individual points, whose evaluation is macroscopic. Each element's position in the landscape, marked by an $\mathrm{x}$, is defined by its $\mathrm{x}$ - and $\mathrm{y}$-coordinate. The focus is on identifying groups of elements and segmenting areas so to provide a visual representation to derive measures from. 


\section{State of Art and Research}

The state of the art covers the relevance of matrices for the problem at hand, applications of DSMs for coordinating projects with regard to market and product perspective as well as suitable criteria for assessing internal effort and customer value of variability in modules and features.

Features in the context of this paper are properties of a product that can be individualized to shape the customer's experience. Examples are size or power output of an engine, but can also include functional aspects such as the wireless protocols in phones.

A tool for the assessment of modules and features from market and product perspective needs to handle large amounts of data from different perspectives. Eppinger and Browning (2012) argue for the Design Structure Matrix (DSM) and the related Domain Mapping Matrix (DMM) as well as the Multi Domain Matrix (MDM) to be a potent choice in this context, as they can store and visualize large amounts of data. With regard to data coming from market and product perspective, a matrix can store both data and how data sets are correlated in a single model. An essential aspect of matrices as a data storage is the ability of computers to execute calculations with the data set. Computers are able to optimize the data and search for patterns. (Eppinger and Browning, 2012)

Both Zhang (2008) and Sun et al. (2010) discuss the application of DSMs in coordinating development efforts of complex products. Zhang (2008) on the ability of a DSM to document dependencies between development processes. A DSM can cluster related processes and optimize the workflow. Sun et al. (2010) expand the focus to include constraints of limited resources and their effect on project schedules. Neither Zhang nor Sun et al. cover the interactions with other domains, which can influence project schedule and chosen priorities. A more holistic approach as presented by Lagerstrom et al. (2016), who expand the DSM method with the hidden structure approach to optimize a company's product portfolio. Each feature is considered and prioritized with regard to its business case. The handling of dependencies between features is covered through DSMs, revealing a hierarchy of features. However, dependency between features gives no indication on the development effort caused by the feature. No approach using DSMs was found in the literature to connect the external market perspective on variability and the internal product perspective on the development effort needed to providing that variability.

To determine this connection, criteria are needed to assess the customer value of variability of a certain feature, e.g. the color. A model covering the attractiveness of product features is the Kano model (Kano et al., 1984). The Kano model differentiates between attractive features, one-dimensional features and basic features, the last of which the customer implicitly expects to be fully implemented (Sauerwein, 2000). Expanding on the cause for an increasing demand for variability, Fuchs and Golenhofen (2019) argue for an increased empathy with individual customers. In addition, they name the industries capability to provide more individualization in products. The individual customer experience has increased in meaning to a manufacturer. In addition, Weiser et al. (2015) identify products being addressed at international markets with varying market regulations and norms, along with varying customer expectations, as a cause for increased variability in products. Norms however form a minimum requirement, which has to be met. There is no flexibility for a 
company trying to enter a market with its product. Factors influencing the longing for variability in a feature, such as color, depend on the customer experience associated with this feature and market expectations (Fuchs and Golenhofen, 2019). The literature review produced no set of criteria to determine when customers do or do not prefer individualization in products.

From a company's perspective, individualization is a cost factor and needs to be accounted for. Creating variability in a module has secondary effects, which can be anticipated using a DMM as shown by Eppinger and Browning (2012). A simple model visualizing the degree of flexibility of a module is the onion peel model. The model consists of the concentric circles of different radius, forming three distinct areas. Changes of modules at the center require a high degree of effort. Flexibility increases with increased distance from the core.

To determine the location within the onion peel model, the flexibility of changing a certain module aspect, such as weight or the number of wheels, has to be assessed. To determine the flexibility, Aleksic (2015) defines a weighted module-change-flexibility-indicator. The suggested dimensions of this indicator include frequency of use, importance to customers, production costs and process costs. Aleksic points out that the dimensions have to be chosen on a case-by-case basis. Adding to the list, Clarkson et al. (2004) point out secondary effects related to the level of dependency towards other module aspects as an important dimension when considering the flexibility. This relates to the product complexity as influence on the design effort as discussed by Bashir and Thomson (2001).

A customer oriented approach to organizing and structuring development projects is the Quality Function Deployment method (QFD). QFD can be structured into four phases, ranging from concept development to deployment and addresses the correlation between what the customer needs and how the company can achieve this goal (Saatweber, 2016). A common tool in this context is the House of Quality (HoQ) method, which also covers a market benchmark as well as the correlations between means of addressing customer needs. HoQ includes means to validate how well a target is met by requiring a quantifiable assessment of each solution aspect (Saatweber, 2016).

Deciding on a scale for qualitative assessments is part of QFD. Patzak and Rattay (2009) suggest a progressive scale of $0,1,3,9$ to spread out the results apart, as opposed to a linear scale of $0,1,2,3,4$. The same progressive scale is deployed by Schuh et al. (2016). Fuchs and Golenhofen (2019) use a linear scale of 0, 1, 3, 5 for QFD, also spreading results apart. No clear recommendation was found as to when deploy which scale for highest efficiency. 


\section{Matrix-Based Landscape Concept}

This section introduces the main concept of the paper. Section 3.1 gives an overview of the matrix-based framework, the domains of which are detailed in section 3.2. Section 3.3 discusses each step of the framework, beginning with an explanation of the matrices involved in obtaining the landscapes. Finally, section 3.4 presents a way to evaluate the two landscapes created in the process.

\subsection{Overview of the Landscape Concept}

The concept centers on the MDM matrix A in Figure 1, which connects the domains Iexternal criteria, II-internal criteria, III-modules and IV-features. Matrix B as a segment of A relates the criteria to the modules and features. Consider a car as an example: module aspects such as drivetrain power are columns of domain III; features such as color are columns of domain IV. Summing up assessments of internal criteria such as engineering efforts and external criteria such as impact on prestige for each column yields the $\mathrm{x}$ - and $\mathrm{y}$ values of its position in the corresponding landscape. Domain III forms the module landscape representing the product perspective; domain IV forms the feature landscape representing the market perspective. Examples of each landscape are shown in Figure 1. The position in the landscape indicates how to prioritize the respective element.

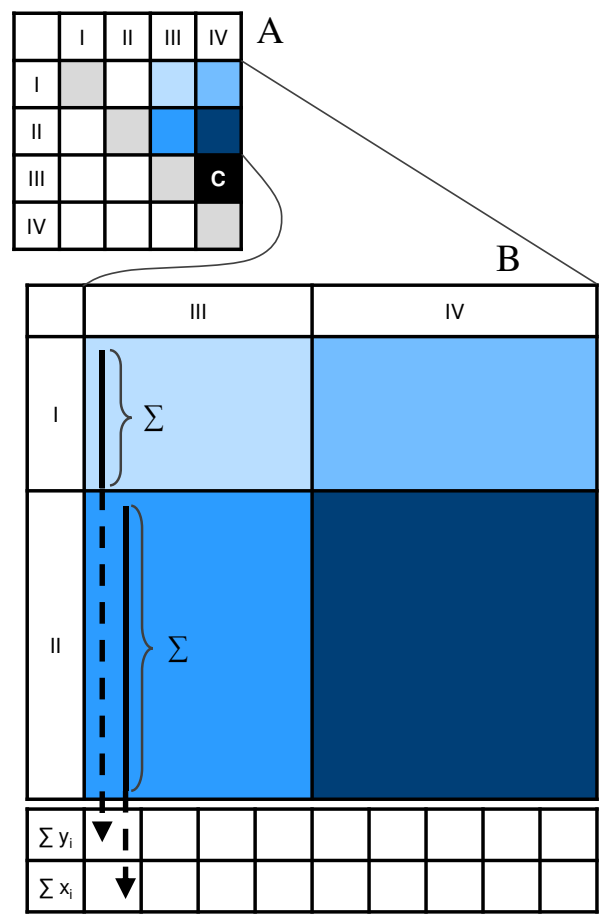

Each $\mathrm{x}$ represents a column of $\mathrm{B}$.

Columns of IV are located in landscape 1, columns of III in landscape 2.
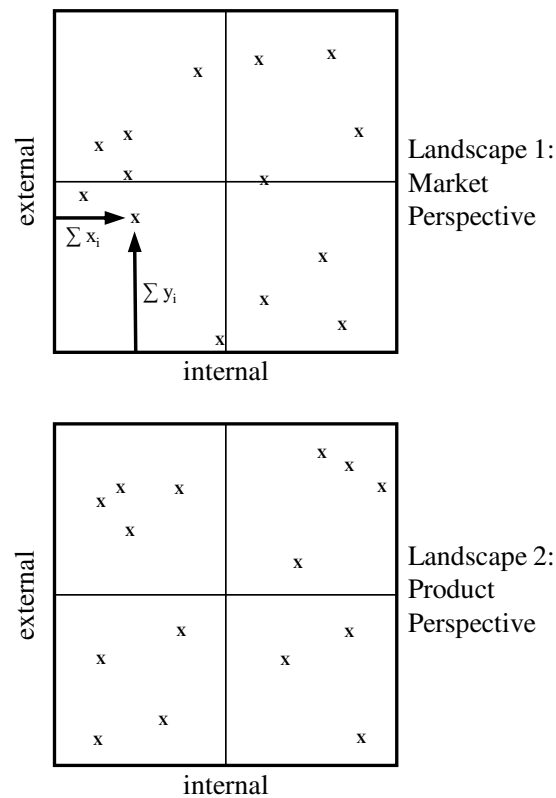

Figure 1. Concept of how to obtain landscapes of the modules and the features from an internal and external perspective. 
The landscapes are the central result of the process. They show for each feature and each module both the appeal of a higher variability from the customer's perspective on the yaxis and the associated effort from an internal perspective on the $\mathrm{x}$-axis. This is a valuable tool for both market and product perspective, as it allows them to identify how their respective object of responsibility affects the other side. The market perspective can determine where offering variability has little impact on product development. These features offer themselves to be promoted as unique selling points and to satisfy customer's needs. The product perspective can determine where reducing variants, cost and complexity is possible without diminishing the products appeal. Again, these are areas to be prioritized when making strategic decisions and planning how to spend the available development resources. Additionally, they lay the groundwork for creating a platform concept or a modular product system concept.

\subsection{Domains and Criteria}

Figure 1 shows how four domains are related to each other in this framework:

I. External Criteria: The influence on how attractive the product is (i.e. ergonomics)

II. Internal Criteria: The influence of increased variability on costs

III. Modules: The product modules or assembly groups (i.e. seat)

IV. Features: Properties relevant to customer individualization (i.e. color)

As discussed in the literature, external criteria to determine the need for variability in a feature have to include both attractiveness of the feature and impact of feature variability on customer experience and customer journey. The literature review yielded no specific criteria to determine how attractive variability of a certain feature is to a customer. Dimensions affecting the customer experience depend on the product. Consider the aforementioned example of a car: the dimensions could include ergonomics, prestige associated with the car, availability and the ecological footprint. While the dimension of prestige may favor a high degree of individualization, the dependence on the cars availability would favor robustness over variety. As the paper aims at prioritizing standardization and individualization of products, it is not useful to consider the norms of a given market as external. The strategic decision of a company to enter the market implies that the product has to meet legal norms. There is no degree of freedom in this dimension.

With regard to internal criteria describing the effort associated with changing a module aspect, the paper takes an approach similar to the onion peel model. As Aleksic (2015) points out, the exact dimensions discussed in section 2 have to be set individually. The degree of dependence on other module aspects is a dimension that can be deducted from the product architecture or interviews with responsible engineers, if the documentation of the architecture is not available. Exemplary dimensions for the assessment are manufacturing costs, development effort and frequency of usage as well as degree of interdependency. Because a quantitative assessment can be resource intensive, a qualitative assessment from the development department is suggested. As for the external criteria, the progressive scale $[0,1,3,9]$ is used. 
Modules as stable elements over product generations compose the structure of the product and represent the first level of decomposition in the product perspective. Module aspects in context of this paper are dimensions of a module that can determine product variants. Depending on the required granularity, the list can remain on the level of modules or expand to assembly groups, both bought and self-developed. It is not useful to include elements below the level of assembly groups, as individual parts such as screws are not relevant for a strategic decision on the product portfolio. The module aspects can be derived from analyzing a company's product portfolio and BOM, or from the documentation of a product's architecture.

\subsection{Application of Matrices}

Starting with the four domains discussed in section 3.2, the MDM matrix A is formed. In the context of this paper, within the internal and the external view we focus on both modules and features. For this purpose matrix B is selected in Figure 1. Matrix B relates internal and external criteria to both modules and features and therefore provides all information to visualize the landscapes.

As discussed in section 2, it is important to spread the scale. Otherwise, a large amount of slightly relevant entries may outweigh a critically important entry. For this purpose, $[0,1,3,9]$ are chosen as possible entries for matrices (I, IV) and (II, III). As a progressive scale, this enhances the spread of results. Filling in all four DMMs (I, III), (I, IV), (II, III) and (II, IV) is no arbitrary task, as only two of them can be easily filled.

Matrix (I, IV) relates external criteria, such as a feature's or module aspect's impact on ergonomics, to features relevant to customers, such as color, and can be filled in from the market perspective. Matrix (II, III) relates internal criteria such as reconstruction effort to modules and can be filled from the product perspective. However, neither market nor product perspective can fill in the remaining two DMMs (I, III) and (II, IV) by themselves. This is where matrix $\mathrm{C}$, seen in Figure 1 as a black matrix, is needed. Matrix $\mathrm{C}$, which is (III, IV) in matrix A, relates modules to features: Each entry of (III, IV) documents how relevant a certain module is for a certain feature. It is also possible to read $\mathrm{C}$ the other way around: How strong is the impact of increased/decreased variability of a certain feature, such as color, on a certain module aspect, such as the car's length? This question has to be answered by an interdisciplinary team consisting of members from both market and product perspective and needs to be revisited as modules change. The scale covers $[0,1 / 2,1]$, representing no impact, slight impact and strong impact. As opposed to the scale of $[0,1,3,9]$ used previously, this scale does not assess the degree to which an element meets a criterion, but rather whether or not two elements are related.

Figure 2 shows how matrix $\mathrm{C}$ is used to calculate (II, IV). Each row of (II, III) is an internal criterion. Each entry in $\mathrm{C}$ is the degree of dependence between a module aspect and a feature. Multiplying (II, III) with C is multiplying the internal criterion's value of each module aspect with that aspects influence on a feature, yielding the internal criterion's impact on the feature. 
B

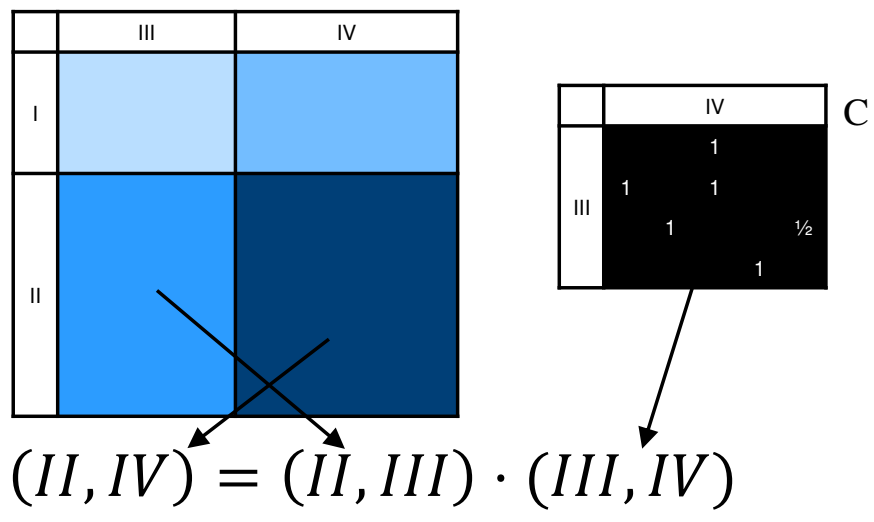

Figure 2. Matrix multiplication yielding (II, IV)

Similarly to the operation in Figure 2, (I, III) can be calculated using $\mathrm{C}^{\mathrm{T}}$, which is (IV, III). Overall, three matrices need to be filled in by the users: (I, IV), (II, III) and (III, IV). A simple programming routine can calculate the remaining entries by matrix multiplication.

With all matrices filled, the $\mathrm{x}$ - and $\mathrm{y}$-values for each landscape can be calculated as shown in Figure 3. First, the sum for each column is calculated for all four DMMs. To cancel out effects from having more or fewer criteria, the values from each DMM are normed. This way, no meaning is implied by any absolute $x$ - or $y$-value from any DMM. This results in $\mathrm{x}$ - and $\mathrm{y}$-values normed the interval $[0,1]$ for each module and each feature. The $\mathrm{x}$-value represents the development effort related to an increase in variability, the y-value the customer satisfaction associated with a high degree of variability. It is the basis for visualizing the two landscapes.

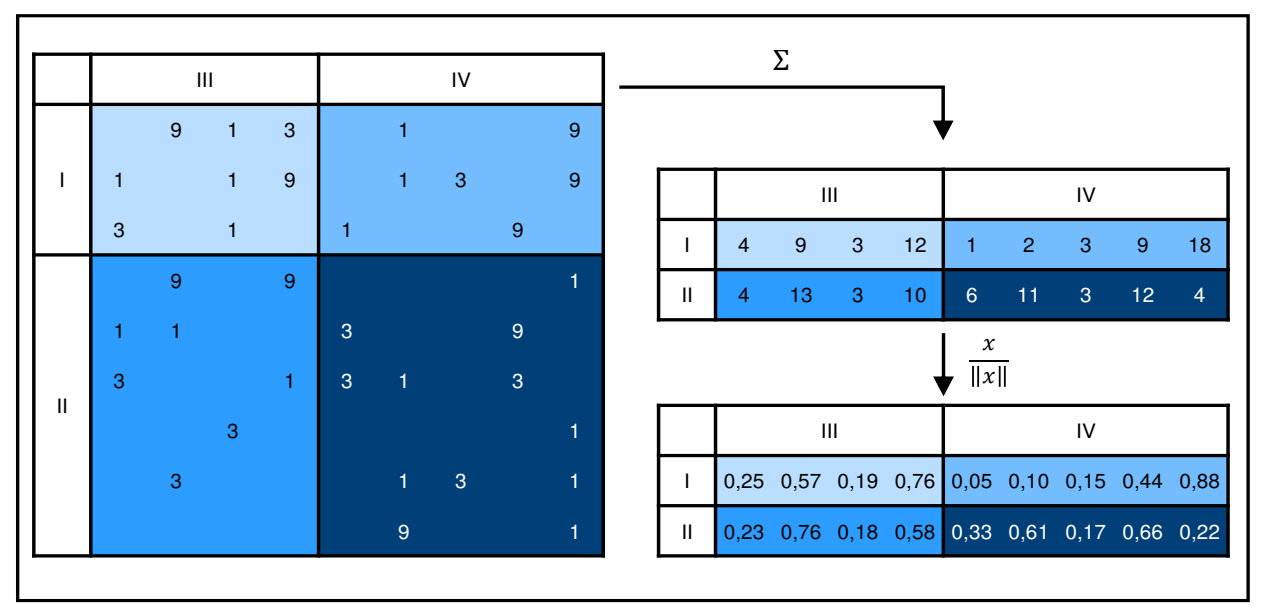

Figure 3. Calculating $\mathrm{x}$ - and y-values from matrix B, empty fields are equal to zero 


\subsection{Evaluation of the Landscapes}

This section discusses how to evaluate the landscapes (Figure 4) resulting from the process described in section 3.3. They appear similar, yet contain different information and address different stakeholders. The feature landscape is directed towards the market perspective and concerns the degrees of freedom when offering individualization to customers. It suggests priorities when drawing up sales strategies and suggestions for optimized product portfolios. The module landscape shows the impact of minimizing costs of certain modules on how attractive the module aspect is to the customer and is directed towards the product perspective. Each landscape supports prioritization when planning resource capacity. Quadrants are suggested to separate areas in the landscapes, though further application may suggest alternative forms of separation.

An $x$ located in quadrant 1 indicates a high degree of impact of variability on customer satisfaction, while the degree of internal cost associated with variability of the module aspect or feature is indicated to be low. These are modules and features, which can be individualized with low resource consumption and should be prioritized. The efficiency of spending resources on them is indicated to be the highest. An x located in quadrant 2 still indicates a high value to the customer, but comes at high internal costs. The effectiveness regarding customer satisfaction of spending resources on variability of such modules and features is high, but the efficiency is low. The market perspective has an incentive to ask for individualization, while the product perspective has an incentive to simplify these entries. An $\mathrm{x}$ located in quadrant 3 suggests low demand for individualization. With resource consumption being low, development has little incentive to reduce variability. These are entries whose priority should be set low, because neither cost savings nor customer satisfaction is to be gained from changing variability.

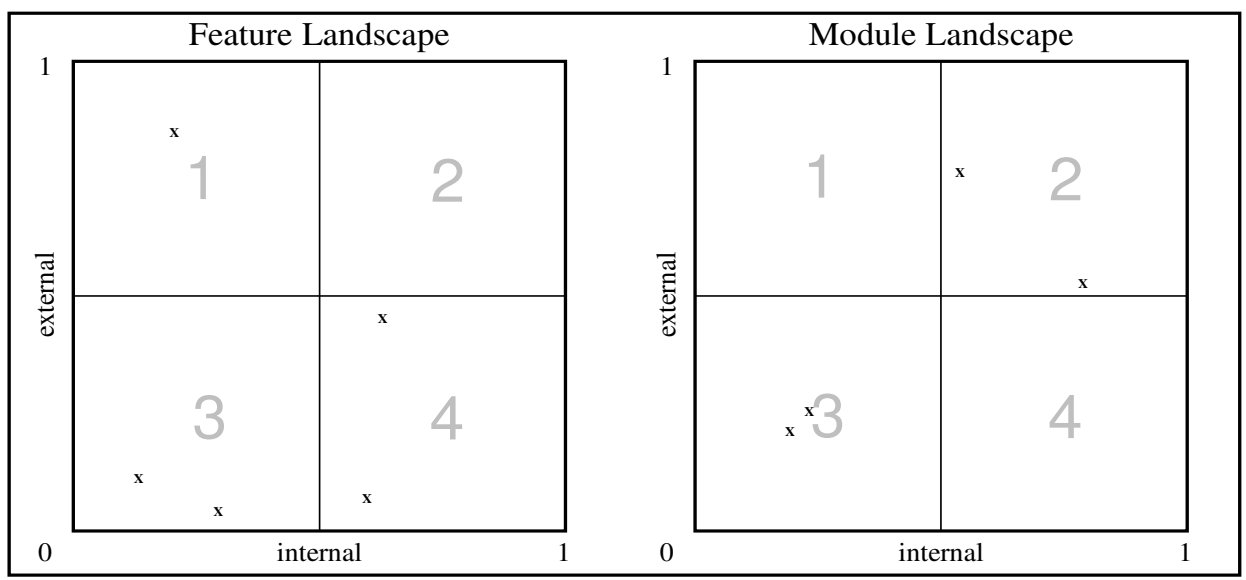

Figure 4. Example landscapes for the modules (left) and the features (right)

An x located in quadrant 4 suggests a minor influence on customer satisfaction through variability, but a large influence on costs. Standardization measures are most efficient for these entries and should be prioritized accordingly. 
Returning to the example of a car, drive train power may be located at medium internal effort and high external attractiveness. Variations in software of the acceleration pedal, giving customers a feeling of a stronger engine, can already produce high satisfaction. Engines can vary without redesigned most aspects of a car. Color is an example of a feature with minimal internal effort and a medium degree of external attractiveness. It allows for individualization without redesigning the hardware. The chassis shape of the car does allow for a strong increase in prestige and customer satisfaction. Individualization and high variance comes at high internal construction efforts, as all aspects of the hardware are impacted; within a car model there would be next to no variation of this aspect.

\section{Conclusion and Outlook}

The paper presents a framework to support product management, sales strategies and development planning by correlating the market perspective with the product perspective. This way, it is possible to specify for each feature and for each module aspect how attractive an increased variety is for the customer and how much effort would be required to offer that variety. The framework uses two DMMs to document the perspectives and a third DMM to document the correlation between features and module aspects. The DMMs need to be filled in and maintained manually. With this input, a program can visualize the two landscapes. A need for future research has been identified in criteria to assess the customer value of increased variability. Although it seems as if it were a fact, that customer's want more individuality, little research was found regarding why this is the case and what specific value the customer hopes to get from individualization. Customized solutions have an increased likelihood of engineering errors, as there is less routine associated with their development. Another field of interest is the segmentation of the two landscapes. Implications of each quadrant have been derived from logical reasoning. Future applications of the landscapes may suggest a different segmentation that leads to conclusions that are more specific to support strategic decisions and balance conflicting interests regarding standardization. Most parts of the matrix A in Figure 1 are irrelevant to the problem at hand, however they may be interesting for solving other challenges. Relating the core features of the product causing variability to each other may be of interest to general variant management, while relating all modules to each other may be of interest to the product architecture. Other than approaches such as QFD and HoQ, the proposed approach is a tool, rather than a development framework. It serves as a facilitator of communication within the existing development process.

\section{Acknowledgement}

The presented results have been developed within the research project "Complexity oriented design of cyber-physical systems" [GZ: SCHU 1495/146-1] funded by the Deutsche Forschungsgemeinschaft (DFG).

\section{References}

Aleksic, S., 2015. Nachhaltige Weiterentwicklung von modularen Produktarchitekturen durch Release-Management. Dissertation, 1st ed. Aachen, 287 pp.

Alptekinoğlu, A., Corbett, C.J., 2008. Mass Customization vs. Mass Production: Variety and Price Competition. M\&SOM 10 (2), 204-217. https://doi.org/10.1287/msom.1070.0155. 
Anderson, S.W., 1995. Measuring the impact of product mix heterogeneity on manufacturing overhead cost. The Accounting Review 70 (3), 363-387.

Bashir, H.A., Thomson, V., 2001. Models for estimating design effort and time. Design Studies 22 (2), 141-155. https://doi.org/10.1016/S0142-694X(00)00014-4.

Clarkson, P.J., Simons, C., Eckert, C., 2004. Predicting Change Propagation in Complex Design. Journal of Mechanical Design 126 (5), 788-797. https://doi.org/10.1115/1.1765117.

Crawley, E., Cameron, B., Selva, D., 2016. System architecture: Strategy and product development for complex systems, 479eiten.

Eppinger, S.D., Browning, T.R., 2012. Design structure matrix methods and applications. MIT Press, Cambridge Mass., xii, 334.

Fuchs, C., Golenhofen, F., 2019. Mastering Disruption and Innovation in Product Management. Springer International Publishing, Cham, 298 pp.

Kano, N., Seraku, N., Takahashi, F., ichi Tsuji, S., 1984. Attractive Quality and Must-Be Quality. Journal of the Japanese Society for Quality Control 14 (2), 147-156.

Lagerstrom, R., Addibpour, M., Heiser, F., 2016. Product feature prioritization using the Hidden Structure method: A practical case at Ericsson, in: 2016 Portland International Conference on Management of Engineering and Technology (PICMET), Honolulu, HI, USA. 04.09.2016 08.09.2016. IEEE, pp. 2308-2315.

Patzak, G., Rattay, G., 2009. Projektmanagement: Leitfaden zum Management von Projekten, Projektportfolios, Programmen und projektorientierten Unternehmen, 5th ed. Linde, Wien, $739 \mathrm{pp}$.

Saatweber, J., 2016. Produkte entwickeln mit QFD - Quality Function Deployment, in: Lindemann, U. (Ed.), Handbuch Produktentwicklung. Hanser, München, pp. 629-671.

Sauerwein, E., 2000. Das Kano-Modell der Kundenzufriedenheit: Reliabilität und Validität einer Methode zur Klassifizierung von Produkteigenschaften. Deutscher Universitätsverlag, Wiesbaden, $259 \mathrm{pp}$.

Schuh, G., 2013. Lean Innovation. Springer Berlin Heidelberg, Berlin, Heidelberg, 255 pp.

Schuh, G., Riesener, M., 2017. Produktkomplexität managen: Strategien - Methoden - Tools, 3rd ed. Hanser, München, 273 pp.

Schuh, G., Riesener, M., Mattern, C., 2016. Approach to evaluate complexity in new product development projects. Int. J. DNE 11 (4), 573-583. https://doi.org/10.2495/DNE-V11-N4-573583.

Sun, Y.-n., Cui, R., Sun, T., 2010. The Resource Constraints Project Scheduling Based on DSM, in: 2010 International Conference on Management and Service Science, Wuhan, China. 24.08.2010 - 26.08.2010. IEEE, pp. 1-4.

Vangheluwe, H., 2018. Multi-paradigm modelling of cyber-physical systems, in: Proceedings of the 4th International Workshop on Software Engineering for Smart Cyber-Physical Systems SEsCPS '18. the 4th International Workshop, Gothenburg, Sweden. 27.05.2018 - 29.05.2018. ACM Press, New York, New York, USA, p. 1.

Weiser, A., Baasner, B., Ovtcharova, J., 2015. Necessity of the consideration of strategic aspects in variant decisions of modular product architectures, in: 2015 IEEE International Conference on Industrial Engineering and Engineering Management (IEEM), Singapore. 06.12.2015 09.12.2015. IEEE, pp. 1551-1555.

Zhang, H., 2008. Product Development Processes Management Based on Design Structure Matrix (DSM) Method: WiCOM '08; Dalian, China, 12.10.2008 - 17.10.2008. IEEE Operations Center, Piscataway, NJ, 4 pp.

Contact: Benjamin Lender, Laboratory for Machine Tools and Production Engineering WZL of the RWTH Aachen University, b.lender@wzl.rwth-aachen.de 